\title{
Acute Flaccid Paralysis: A Registry-based Study of Demographic, Clinical, and Diagnostic Characteristics of Children in a Referral Center in Iran
}

\author{
Ali Reza Tavasoli ( $\sim$ a_tavasoli@sina.tums.ac.ir ) \\ Tehran University of Medical Sciences \\ Roxana Pazouki \\ Tehran University of Medical Sciences \\ Sareh Hosseinpour \\ Tehran University of Medical Sciences \\ Mahmoud Reza Ashrafi \\ Tehran University of Medical Sciences \\ Masood Ghahvechi Akbari \\ Tehran University of Medical Sciences
}

Sanaz Rezaei

Shaheed Beheshti University of Medical Sciences

Nima Parvaneh

Tehran University of Medical Sciences

Mohammad Vafaee-Shahi

Tehran University of Medical Sciences

Bahram Mohammadi

Tehran University of Medical Sciences

Alireza Abdi

Tehran University of Medical Sciences

Sayna Bagheri

Tehran University of Medical Sciences

Research article

Keywords: Acute, Flaccid, Paralysis, Children, Etiology, Diagnosis

Posted Date: August 19th, 2020

DOI: https://doi.org/10.21203/rs.3.rs-57505/v1 
License: (c) (i) This work is licensed under a Creative Commons Attribution 4.0 International License. Read Full License 


\section{Abstract}

Background: Acute Flaccid Paralysis(AFP) is a clinical syndrome characterized by acute progressive weakness in limbs and respiratory and swallowing muscles. Etiologies of AFP mostly include infections in anterior horn cells of the spinal cord, Guillain-Barre syndrome(GBS), transverse myelitis, viral myositis, myasthenia gravis and botulism. An accurate and early diagnosis of AFP is crucial. The aims of this study were to determine the main etiologies and characteristics of AFP in children and also design a diagnostic approach for AFP based on a hospital-based registry system.

Method: This retrospective, cross-sectional study is a five-years survey, from 2011 to 2016 in Children's Medical Center as the main children referral hospital in Iran. Totally 118 patients who had met AFP ICD10- $\mathrm{CM}$ and inclusion criteria were included. All clinical and Paracinical data were collected based on a prepared questionnaire for the study.

Result: The mean age of the patients was $6.09 \pm 3.60$ years old. The most frequent underlying etiologies were GBS, viral myositis and Vaccine-Associated Paralytic Poliomyelitis(VAPP) accounting for $67.8 \%$, $15.3 \%$ and $5 \%$ of the patients, respectively. In terms of preceding clinical symptoms coryza, fever and gastrointestinal symptoms were more common. The most common neurologic presenting symptom was motor dysfunction. Cerebrospinal fluid analysis mainly indicated increase level of protein in favor of GBS. Elevated Creatine kinase level with the mean of $2184 \mathrm{IU} / \mathrm{L}$ has been reported in patients with viral myositis. Brain imaging principally revealed normal results and spinal MRI findings were compatible with the diagnoses of GBS and transvers myelitis. All stool sample results for polio virus detection were negative.

Conclusion: In this study, we determined the etiologies of AFP in Iranian children referred to Children's Medical Center. Guillain-Barre syndrome, viral myositis, transverse myelitis and VAPP were the most common etiologies. We also recommended a diagnostic approach for AFP in pediatric population regards to main etiologies in emergency room of a children referral hospital.

\section{Background}

Acute Flaccid Paralysis (AFP) is a complex clinical syndrome in children below 15 years of age, characterized by sudden onset of weakness in one or more limbs and weakness of the respiratory and swallowing muscles, progressing to the maximum severity within several days to weeks ${ }^{1}$. The term "flaccid" is defined as the absence of spasticity, hyperreflexia, clonus, or extensor plantar response due to the Central Nervous System (CNS) motor tract disorders, but not trauma or transient causes such as postictal phase of acute seizure ${ }^{2}$.

A broad array of etiologies can give rise to AFP such as infections in anterior horn cell of the spinal cord [poliomyelitis, non-polio enterovirus, Vaccine-Associated Paralytic Poliomyelitis (VAPP), Guillain-Barre syndrome (GBS), transverse myelitis, viral myositis, myasthenia gravis and botulism] ${ }^{3-4}$. World Health Organization (WHO) regularly collects data on the incidence of AFP in children younger than 15 years as 
a part of the Global Polio Eradication Initiative (GPEI). In this regard, they witnessed a dramatic decline in polio incidence over the last two decades around the world when people started to be routinely

vaccinated against the virus; so, nowadays, GBS has replaced polio as the most common cause of AFP 5 .

Nevertheless, several studies have been conducted in various cities and provinces of Iran during the last 25 years, suggesting the most common underlying etiology of AFP as GBS, in agreement with the findings of most of other countries and except for one case of polio in 1995, no such diagnosis was detected ${ }^{7-9}$.

Main etiology of AFP in each patient can be precisely diagnosed through its exclusive clinical and para clinical symptoms and signs. Since untreated AFP could lead to death through respiratory muscle failure, accurate and early diagnosis and treatment of the underlying disorder appears mandatory ${ }^{1}$. Accordingly, having a specific diagnostic approach for the management of AFP in the emergency department is required.

In the first step, we determined the main etiologies of AFP in children who referred to the emergency room of Children's Medical Center, which has been known as the main referral hospital for admission of children across the country during the period between 2011 and 2016. During the next step, we designed a diagnostic approach for AFP, which is appropriate for children. To the best of our knowledge, this hospital-based investigation provides help for approaching AFP in children younger than 15 , and can bring about further and better outcome in patients.

\section{Methods}

\section{2-1. Study design and setting}

This descriptive, cross-sectional, retrospective study was performed based on the documented records survey and ICD-10-CM based hospital registry system during 2011 to 2016 at the Children's Medical Center (CMC), as the largest pediatric referral hospital in the country is affiliated to Tehran University of Medical Sciences (TUMS). The study population was the patients who had been referred to the CMC with AFP manifestation. This study protocol was approved by ethical committee of TUMS (Grant Number: IR.TUMS.MEDICINE.REC.1396.3122). Of course, the aims of the study were completely described and written consents were taken from patients' parents or their legal guardians.

\section{2-2. Participants}

We enrolled children who met the criteria for AFP diagnosis according to the International Classification of Diseases, 10th Revision, Clinical Modification (ICD-10-CM). Children whose parents were not cooperative for either adhering to their children's medical treatments or sharing their documents were excluded. Other exclusion criteria were the presence of ataxic gait, the history or current diagnosis of transient weakness such as post-ictal motor weakness, acute spastic paralysis, presence of uncontrolled 
seizures, history of routinely receiving medication, history of recent trauma, history of drug abuse within the last 6 months, and presence of any other medical problems. Parent or legal guardians were informed that they are free to withdraw from the study without any adverse effect on the treatment process of their children.

\section{2-3. Data collection}

We retrospectively included information regarding demographic characteristics, health status, presenting clinical symptoms and signs, laboratory data, neuroimaging modalities, and administration information. Clinical symptoms were categorized into non-neurological symptoms such as fever, coryza, diarrhea, nausea and vomiting, and myalgia while neurological symptoms were mainly related to motor system, sensory impairment, autonomic abnormalities of the nervous system such as bowel and bladder dysfunctions, meningism symptoms, and anterior horn cell damage symptoms like fasciculation. Furthermore, in terms of neurological examination, available information on the strengths of muscles, autonomic dysfunction-related symptoms, deep tendon reflexes, and cranial nerve examination were extracted from the database.

Moreover, we included electrophysiological study's findings such as Electromyography (EMG) and Nerve Conductive Velocity (NCV) that had been conducted in patients with lower motor neuron signs or symptoms to differentiate neuromuscular disorders such as demyelinating neuropathy, axonal neuropathy, neuromuscular junction disorder, preganglionic lesion, myopathy, and anterior horn cell disorders.

We evaluated the data related to stool exams in the patients since two stool samples 24-48 hours apart within 14 days since the onset of paralysis should be collected from all of the AFP patients to exclude involvement with the Poliovirus. Basic biochemical lab findings (CBC, ESR, CPK, Aldolase) were collected. Cerebrospinal Fluid (CSF) analysis was also taken into consideration to determine other viral or bacterial infections. Data on neuroimaging modalities including Computed Tomography (CT) and Magnetic Resonance Imaging (MRI), which had been executed in AFP patients with possibility of CNS involvement were also added to our analyses.

\section{2-4. Statistical analysis}

The data was analyzed using the IBM SPSS Version 25 software. In order to describe the quantitative variables, central indicators such as mean and median measurements, dispersion indices such as standard and amplitude deviation, and to describe qualitative variables, indices such as frequency percentage have been used.

\section{Results}




\section{3-1. Demographic data and AFP etiologies}

During the five-year survey, from 2011 to 2016, among 641 patients who had met AFP ICD-10- CM criteria, 118 cases were included in this study by considering exclusion criteria. From among118 patients, 77 cases $(65.3 \%)$ were boys and 41 (34.7\%) were girls, and the mean age of the patients was $6.09 \pm 3.60$ years old. The most frequent underlying etiologies were Guillain-Barre Syndrome and viral myositis accounting for $67.8 \%$ and $15.3 \%$ of the patients, respectively. As evident, no confirmed case of wild type poliomyelitis was observed. We had 6 cases of VAPP, all immune deficient with diagnosis of X-linked agammaglobulinemia (XLA) in three cases, Severe Combined Immune Deficiency (SCID) in two, and Common Variable Immunodeficiency (CVID) in one case. The prevalence of different etiologies of AFP in children is described in Table 1.

Table 1

The prevalence of different etiologies of AFP.

\begin{tabular}{|lll|}
\hline Diagnosis & Frequency & Percentage \\
\hline Guillain-Barre Syndrome & 80 & 67.8 \\
\hline Viral myositis & 18 & 15.3 \\
\hline Transverse Myelitis & 6 & 5.1 \\
\hline VAAP & 6 & 5.1 \\
\hline Botulism & 3 & 2.5 \\
\hline Periodic Paralysis & 2 & 1.7 \\
\hline Inflammatory Myopathy & 1 & 0.8 \\
\hline Spinal mass lesion & 1 & 0.8 \\
\hline Viral Meningitis & 1 & 0.8 \\
\hline Total & $\mathbf{1 1 8}$ & $\mathbf{1 0 0}$ \\
\hline
\end{tabular}

\section{3-2. AFP manifestations}

In terms of systemic clinical symptoms, $55.9 \%$ had coryza, $39 \%$ of patients had fever, and $15.3 \%$ had gastrointestinal symptoms (i.e. diarrhea, vomiting). Neurologic symptoms evaluation showed motor symptoms in $94.9 \%$, autonomic dysfunction-related symptoms in $37.3 \%$ [pain (82\%), sphincter dysfunction (14\%) and palpitations (2\%)], sensory symptoms in $9.3 \%$, and in addition altered behavior included irritability and mood changes in $2 \%$ of patients. In general, the most common of neurologic 
symptoms was motor problem and among the autonomic symptoms in GBS patients, pain was most commonly observed.

Findings in neurologic examinations included motor involvement in 105 cases (89\%) that was divided to generalized motor involvement in 56 cases (47.5\%), lower limb involvement in 48 patients $(40.7 \%)$, pure upper limb involvement in one case $(0.8 \%)$. Decreased deep tendon reflexes detected in 100 patients (84.7\%) were further subdivided in $46.6 \%$ with lower limb involvement, $37.3 \%$ with both upper and lower limb, and $0.8 \%$ with pure upper limb involvement.

Based on the patients' medical records, the average duration between onset of acute flaccid paralysis manifestation and hospitalization was 4 days. The prevalence of AFP manifestations was more common in winter and spring. In AFP patients with GBS clinical impression, IVIG therapy started in average one day before the diagnosis confirmation.

\section{3-3. Diagnostic tools}

CSF analysis of 69 patients who underwent a Lumbar Puncture (LP) demonstrated that the mean level of protein was 74 (63.8-84-2), and the mean count of CSF WBC count and glucose level were 5 (3.6-6.4) and 61 (59.4-62.6) respectively, which mainly indicated an increase in the level of protein in CSF analysis. In addition, the CSF analysis of 57 out of these 69 patients showed only an increase in CSF mean protein level of 78 (68-88) $\mathrm{mg} / \mathrm{dl}$, which was more in favor of GBS diagnosis.

Elevated mean CPK level of $2184 \mathrm{IU} / \mathrm{L}$ has been reported in 18 patients, in favor of the diagnosis of viral myositis whereas CPK levels were within normal limit in other patients.

In total, 79 patients including 65 cases of GBS underwent EMG and NCV, and the most common finding was demyelination neuropathy pattern. The mean level of NCV was 38 (36.4-39.6) m/s which was lower than normal limit (Supplementary table 1).

Thirty patients underwent brain CT scans and all were normal. In addition, 16 patients underwent a spinal MRI. The spinal MRI of 5 patients showed surface thickening and marked enhancement in the anterior spinal cord nerve roots, particularly in the region of the conus medullaris and cauda equine which were in favor of the diagnosis of GBS. In the spinal MRI of 5 patients, increased T2-W signal changes were also detected in variable size and extension which was in favor of transverse myelitis. Nevertheless, 5 patients had a normal spinal MRI report, but only one patient had a spinal mass lesion in lumbosacral region which revealed a spinal Astrocytoma following further evaluation. The results of the reports of 2 out of 14 patients who underwent a brain MRI revealed non-specific findings including generalized cerebral atrophy and non-specific deep white matter signal intensities.

As for laboratory tests, two stool samples were collected in $80 \%$ of cases; then fecal specimen was analyzed by the reference laboratory in order to detect poliovirus, with negative results in all cases. $85 \%$ of the patients had normal stool exam results, and $15 \%$ had non-specific abnormal results. 


\section{Discussion}

In this cross-sectional study, a total of 118 patients with clinical presentation of AFP were assessed with regards to demographic, clinical and diagnostic data and final diagnosis.

In our study, the average age of patients was $6.09 \pm 3.60$ years old which was higher than most of the other study results. $44.9 \%$ of cases were between $5-10$ years old and $37.3 \%$ of them were under 5 years old, whereas the most common age in children for AFP had been reported under 5 years old in other researches ${ }^{3-6-7-8-9-10}$. Sex differences was according to the previous studies, as well as systemic and neurologic manifestation and examination findings ${ }^{1-6-7-9-10}$.

In the present study, the most four common causes of AFP were included: Guillain-Barre syndrome, viral myositis, transverse myelitis and VAPP. Guillain-Barre syndrome was the most common etiology similar to previous reports ${ }^{1-2-6-8-10}$. Regarding Guillain-Barre syndrome as the most common cause of AFP and in respect of its treatable nature, it seems that good prognosis could be expected in most cases of AFP in children. In addition, with regards to the normal results of brain imaging in the majority of patients, we can conclude that it is not necessary to consider neuroimaging in the initial steps of approach to AFP, and implementing it can be postponed depending on the patient's clinical scenario.

Moreover, it's important to note that the prevalence of AFP was more common in the second half of the year, which can be due to the prevalence of respiratory infections and their role in the onset of the specific diseases such as GBS ${ }^{1-2}$.

In terms of other causes of AFP, some inconsistent findings were noticeable in comparison with previous studies. Viral myositis was found as the second etiology of AFP in our study that was in contrast with other studies which mentioned transverse myelitis as the second common etiology for AFP 6-9-11. However, it should be noted that viral myositis was not included in the list of major causes of AFP in other childhood studies. With regard to the significant prevalence of viral myositis in the present study, the necessity of being more attentive to this entity should be considered in clinical approach to AFP in children. Since some patients with the diagnosis of viral myositis had no pain, with only symptoms of paralysis, it would be suitable to consider the assessment of muscle breakdown biomarkers such as serum CPK and aldolase levels during diagnostic process of AFP in children ${ }^{7-9}$.

Furthermore, apart from our study, there has been no report of VAPP in other researches carried out in Iran for two probable reasons: first, our study was performed in a large pediatric referral hospital which serves as the scientific headquarters of the country, and second, better diagnostic equipment and facilities such as immune deficiency research center in this hospital made this detection possible 6-10-12-13.

In addition, no case of traumatic neuritis was found in this study, whereas in some studies it was mentioned as one of the potential common causes ${ }^{6-14}$. This should be clarified that children with 
traumatic injuries are not usually referred to CMC hospital.

In the present study, no case of poliomyelitis was detected, compatible with all studies since 1995 on AFP in children, the date of last report of polio in Iran. However, several Middle Eastern countries still stand as high-risk regions regarding poliomyelitis because of the lack of mass vaccination and migration trend among those countries ${ }^{6-15}$. Except for a study in Iraq that reported several polios (the last one dating back to the year 2000), in the researches in other countries no case of polio was reported ${ }^{3-12-14}$. It is necessary to mention that sending stool sampling to investigate poliovirus has been done according to the WHO standard guidelines, but the reason that the results of stool investigation only existed for $80 \%$ of patients was a limitation in this study due to issues such as early discharge of the patient, non-polio diagnosis before hospitalization, inability to have the sample due to constipation and not informing the referral health center for stool sample for poliomyelitis.

Finally, based on the present study, we designed a diagnostic algorithm to approach children with AFP in the emergency room. This algorithm is used to improve clinical decision making by planning more assessments, treatment strategies and outcome of treatment. It should be mentioned that detection of poliovirus by stool sampling is recommended for all AFP patients according to WHO guidelines. The algorithm presents a step by step approach to a child by considering AFP etiologies. At first, central nervous system (CNS) associated causes should be ruled out through signs of CNS involvement, and if it is negative, a test for intoxication would be required in the next step. If all these steps are negative, a clinical suspicion of Peripheral Nervous System (PNS) or spinal cord involvement will be raised, and consequently more related investigations to confirm the diagnosis are obligatory (figure 2).

\section{Conclusion}

In this study, we determined the etiologies of AFP in Iranian children who referred to Children's Medical Center hospital. Guillain-Barre syndrome, viral myositis, transverse myelitis and VAPP were the most common etiologies. We also recommend a diagnostic approach for AFP in pediatric population with regards to main etiologies to improve clinical decisions in a large referral children hospital.

\section{List Of Abbreviations}

AFP: Acute Flaccid Paralysis

GBS: Guillain Barre Syndrome

VAAP: Vaccine-Associated Paralytic Poliomyelitis

CNS: Central Nervous System

PNS: Peripheral Nervous System

WHO: World Health Organization 
GPEI: Global Polio Eradication Initiative

TUMS: Tehran University of Medical Sciences

ICD-10-CM: International Classification of Diseases, 10th Revision, Clinical Modification

EMG: Electromyography

NCV: Nerve Conductive Velocity

CBC: Complete blood count

ESR: Erythrocyte Sedimentation Rate

CPK: Creatine phosphokinase

CSF: Cerebrospinal Fluid

CT: Computed Tomography

MRI: Magnetic Resonance Imaging

XLA: X-linked agammaglobulinemia

SCID: Severe Combined Immune Deficiency

CVID: Common Variable Immunodeficiency

LP: Lumbar Puncture

CMC: Children's Medical Center

\section{Declarations}

\section{Ethics approval and consent to participate:}

Institutional Review Board (IRB) and ethical committee of TUMS approved our study (Grant Number: IR.TUMS.MEDICINE.REC.1396.3122).

\section{Consent for publication:}

Informed consent was written by parents of our patient to participate in this study.

\section{Availability of data and materials:}


The datasets analyzed during the current study are available from the corresponding author on reasonable request.

\section{Competing interests:}

The authors declare that they have no competing interests

\section{Funding:}

Not applicable

\section{Authors' contributions:}

Ali Reza Tavasoli participated in the design of the study, interpretation of the data and drafted the manuscript and, also participated in critical review of final manuscript. Roxana Pazouki, Sayna Bagheri, Sanaz Rezaei and Sareh Hosseinpour drafted the manuscript. Masood Ghahvechi Akbari, Nima Parvaneh and Mohammad Vafaee-Shahi participated in diagnosis the patient. Mahmood Reza Ashrafi participated in the acquisition and interpretation of data. Sareh Hosseinpour, Bahram Mohammadi and Alireza Abdi participated in clinical examination and acquisition of data. Roxana Pazouki also participated in taking informed consent.

\section{Acknowledgements:}

We thank our patient's family for participating to our study.

\section{References}

1. Marx A, Glass JD, Sutter RW. Differential diagnosis of acute flaccid paralysis and its role in poliomyelitis surveillance. Epidemiologic reviews. 2000;22(2):298-316.

2. Desai S, Smith T, Thorley BR, Grenier D, Dickson N, Altpeter E, et al. Performance of acute flaccid paralysis surveillance compared with $\mathrm{W}$ orld $\mathrm{H}$ ealth $\mathrm{O}$ rganization standards. Journal of paediatrics and child health. 2015;51(2):209-14.

3. Messacar K, Schreiner TL, Van Haren K, Yang M, Glaser CA, Tyler KL, et al. Acute flaccid myelitis: a clinical review of US cases 2012-2015. Annals of neurology. 2016;80(3):326-38.

4. Hopkins SE. Acute flaccid myelitis: etiologic challenges, diagnostic and management considerations. Current Treatment Options in Neurology. 2017;19(12):48.

5. Craig AT, Butler MT, Pastore R, Paterson BJ, Durrheim DN. Acute flaccid paralysis incidence and Zika virus surveillance, Pacific Islands. Bulletin of the World Health Organization. 2017;95(1):69. 
6. Faheem MU, Haroon MZ, Khan AA, Shaukat M, Anwar SA. ACUTE FLACCID PARALYSIS SURVEILLANCE: A 5 YEARS STUDY OF BANNU, PAKISTAN. Journal of Ayub Medical College Abbottabad. 2015;27(3):673-6.

7. Ali AM, Abdolhussein S. An epidemiological analysis of acute flaccid paralysis in Khuzestan Province, southwest Iran, from 2006 to 2010. Epidemiology and Health. 2016;38(1):10-0.

8. Poorolajal J, Ghasemi S, Farahani LN, Hosseini AS, Bathaei SJ, Zahiri A. Evaluation of acute flaccid paralysis in hamadan, Iran from 2002 to 2009. Epidemiology and Health. 2011;33.

9. Soltani J, Esmailnasab N, Roshani D, Karimi M, Amjadi M-J. Acute flaccid paralysis and its differential diagnosis in in kurdistan province, Western Iran; an 11-year surveillance. Iranian journal of pediatrics. 2014;24(2):131.

10. Landaverde JM, Trumbo SP, Danovaro-Holliday MC, Cochi SE, Gandhi R, Ruiz-Matus C. Vaccineassociated paralytic poliomyelitis in the postelimination era in Latin America and the Caribbean, 1992-2011. The Journal of infectious diseases. 2014;209(9):1393-402.

11. Kong NCftCoWPEiH. Fifteen years of acute flaccid paralysis surveillance in $\mathrm{H}$ ong $\mathrm{K}$ ong: Findings from 1997 to 2011. Journal of Paediatrics and Child Health. 2014;50(7):545-52.

12. Samollovich E, Ermolovich M, Kotova I, Svirchevskaia E, Shimanovich V, Kozhemiakin A, et al. Surveillance of acute flaccid paralysis in Belarus. Zhurnal mikrobiologii, epidemiologii, $\mathrm{i}$ immunobiologii. 2007(2):24-31.

13. Nanteza MB, Kisakye A, Ota MO, Gumede N, Bwogi J, Institute EPolLTUVR. Vaccine associated paralytic poliomyelitis cases from children presenting with acute flaccid paralysis in Uganda. Journal of Medical Virology. 2015;87(12):2163-7.

14. Jasem JA, Marof K, Nawar A, Khalaf Y, Al-Hamdani F, Ali S, et al. An epidemiological analysis of acute flaccid paralysis and its surveillance system in Iraq, 1997-2011. BMC infectious diseases. 2014;14(1):1-9.

15. Davarpanah M, Bakhtiari H, Mehrbani D, KHADEM AF. A 12-years surveillance of poliomyelitis and acute flaccid paralysis in Fars Province, Southern Iran. 2008.

16. Organization, W. H. WHO vaccine-preventable diseases: monitoring system Department of vaccines and biologicals, World Health Organization: 2009

17. McKhann G, Cornblath D, Griffin J, Ho T, Li C, Jiang Z, et al. Acute motor axonal neuropathy: a frequent cause of acute flaccid paralysis in China. Annals of neurology. 1993;33(4):333-42.

18. D'Errico MM, Barbadoro P, Bacelli S, Esposto E, Moroni V, Scaccia F, et al. Surveillance of acute flaccid paralysis in the Marches region (Italy): 1997-2007. BMC infectious diseases. 2008;8(1):1-7.

19. Pellegrinelli L, Primache V, Fiore L, Amato C, Fiore S, Bubba L, et al. Surveillance of acute flaccid paralysis (AFP) in Lombardy, Northern Italy, from 1997 to 2011 in the context of the national AFP surveillance system. Human vaccines \& immunotherapeutics. 2015;11(1):277-81.

\section{Figures}


Acute motor weakness/abnormal gait $\leq 2$ weeks

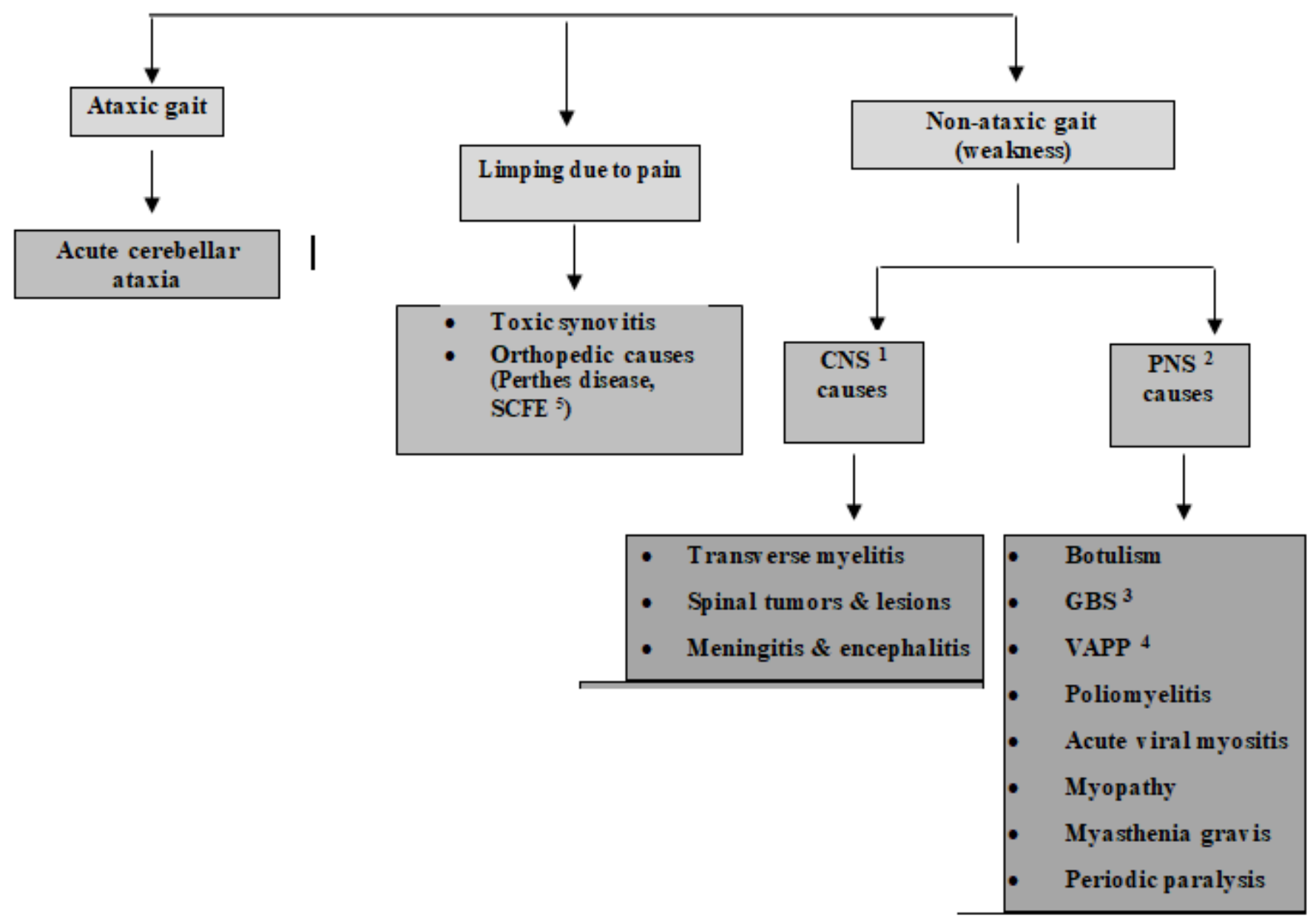

\section{Figure 1}

Display the flowchart used to select patients to enter the study, 1. CNS: Central Nervous System 2. PNS: Peripheral Nervous System 3. GBS: Guillain-Barre Syndrome 4. VAPP: Vaccine-Associated Paralytic Poliomyelitis. 5. SCFE: Slipped Capital Femoral Epiphysis 


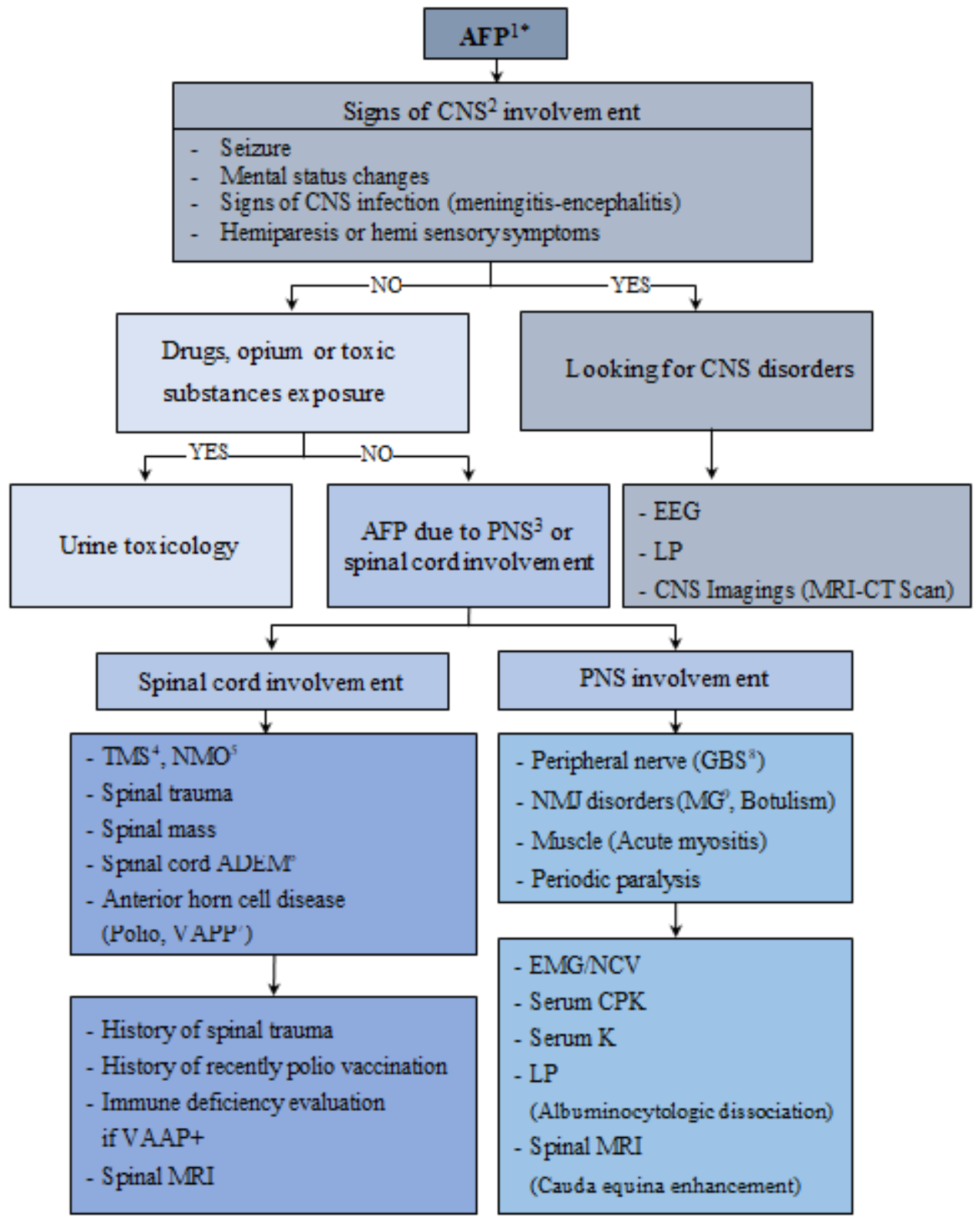

\section{Figure 2}

Recommended diagnostic approach to childrens presenting with AFP. 1. AFP: Acute flaccid Paralysis * Collecting two Stool specimens is recommended by WHO to detect poliovirus at the time onset of AFP 2. CNS: Central Nervous System 3. PNS: Peripheral Nervous System 4. TMS: Transverse Myelitis Syndrome 5. NMO: Neuromyelitis Optica 6. ADEM: Acute Disseminated Encephalomyelitis 7. VAPP: VaccineAssociated Paralytic Poliomyelitis 8. GBS: Guillain-Barre Syndrome 9. MG: Myasthenia Gravis.

\section{Supplementary Files}

This is a list of supplementary files associated with this preprint. Click to download.

- supplementaryTable1.AFP.docx 